\title{
Comparative analysis of yeast species identification using phenotypic methods and real-time PCR
}

\author{
Priputnevich T.V. ${ }^{1}$, Muravieva V.V. ${ }^{1}$, Gordeev A.B. ${ }^{1}$, Lyubasovskaya L.A. ${ }^{1}$, Rodchenko J.V. ${ }^{1}$, \\ Ogneva L.V. ${ }^{1}$, Donnikov A.E. ${ }^{1}$, Kirillov M.Y. ${ }^{2}$, Dubodelov D.V. ${ }^{1}$, Trofimov D.Y. ${ }^{1}$, Sukhikh G.T. ${ }^{1}$
}

${ }^{1}$ National Medical Research Center for Obstetrics, Gynecology and Perinatology named after Academician V.I. Kulakov of Ministry of Healthcare, Moscow, Russia

${ }^{2}$ Company DNA-Technology LLC, Moscow, Russia

http://ncagip.ru/en E-mail: priput1@gmail.com

\section{Objectives:}

At present there is a trend to increase the role of fungi in the development of life-threatening infections. With the dominance of the role of fungi in the development of infectious diseases the role of less common opportunistic yeasts such as Candida nonalbicans, Pichia, Rhodotorula, Trichosporon, Saccharomyces, Malassezia increases. Monitoring of species composition of yeasts and their resistance to antimycotics has not lost its relevance. Fast identification and knowledge of species-specific sensitivity to antimycotics determine the tactics of treatment of patients with mycotic infections. Therefore it is important to use methods of fast species identification. The aim of the study was to compare the results of species identification of clinically significant yeast using phenotypic methods and real-time PCR.

\section{Methods:}

A total of 92 isolates of yeast from vaginal discharge of women of reproductive age and from different loci of infants, and 91 samples of biological materials, obtained microbiological confirmation of the presence of yeast were observed. Inoculation on HiCrome Candida agar (Himedia, India), chlamydospore and hyphal tests were used to differentiate Candida albicans ( $C$. albicans) from non-albicans species during phenotypic identification. Species identification of non-albicans species was conducted using VITEK 2 Compact (bioMerieux, France) and matrix-assisted laser desorbtion/ionization time-of-flight mass spectrometer (MALDI TOF MS) Autoflex III (Bruker Daltonics, Germany). Alternative yeast identification was conducted by real-time PCR with species-specific primers for Candida albicans, C. dubliniensis, C. glabrata, C. tropicalis, C. kefyr, Saccharomyces cerevisiae, Malassezia furfur.

\section{Results:}

For C. albicans identification among isolates and samples of biological materials the results were the same in $98.7 \%$ (77 of 78 ) and $94.8 \%$ (72 of 76 ) cases respectively. In two samples the identification mistakes were related to Candida dubliniensis ( $C$. dubliniensis) which was inaccurate identified as $C$. albicans, and it was identified as $C$. dubliniensis using real-time PCR.
The identification using MALDI TOF MS and sequencing of the 26S rDNA gene confirmed that they were $C$. dubliniensis. Yeasts were not found in 3 of 76 samples by real-time PCR. For identification of non-albicans species (Candida glabrata, Candida tropicalis, Candida kefyr, Saccharomyces cerevisiae, Malassezia furfur) among isolates and samples of biological materials the results were the same in $100.0 \%$ (14 of 14) and $93.3 \%$ (14 of 15) cases respectively. In one sample of biological materials $M$. furfur was isolated by cultural analysis, but it was not found using real-time PCR. Total in 4 samples yeasts were not found by real-time PCR, but they were isolated by cultural analysis in low titer (1-3 lg CFU/ml).

\section{Conclusion:}

Thus, the study revealed a high degree of coincidence of results for yeast identification using phenotypic methods and real-time PCR. It is found both for pure yeast cultures and for samples of biological materials reducing the time of obtaining the results of an analysis.

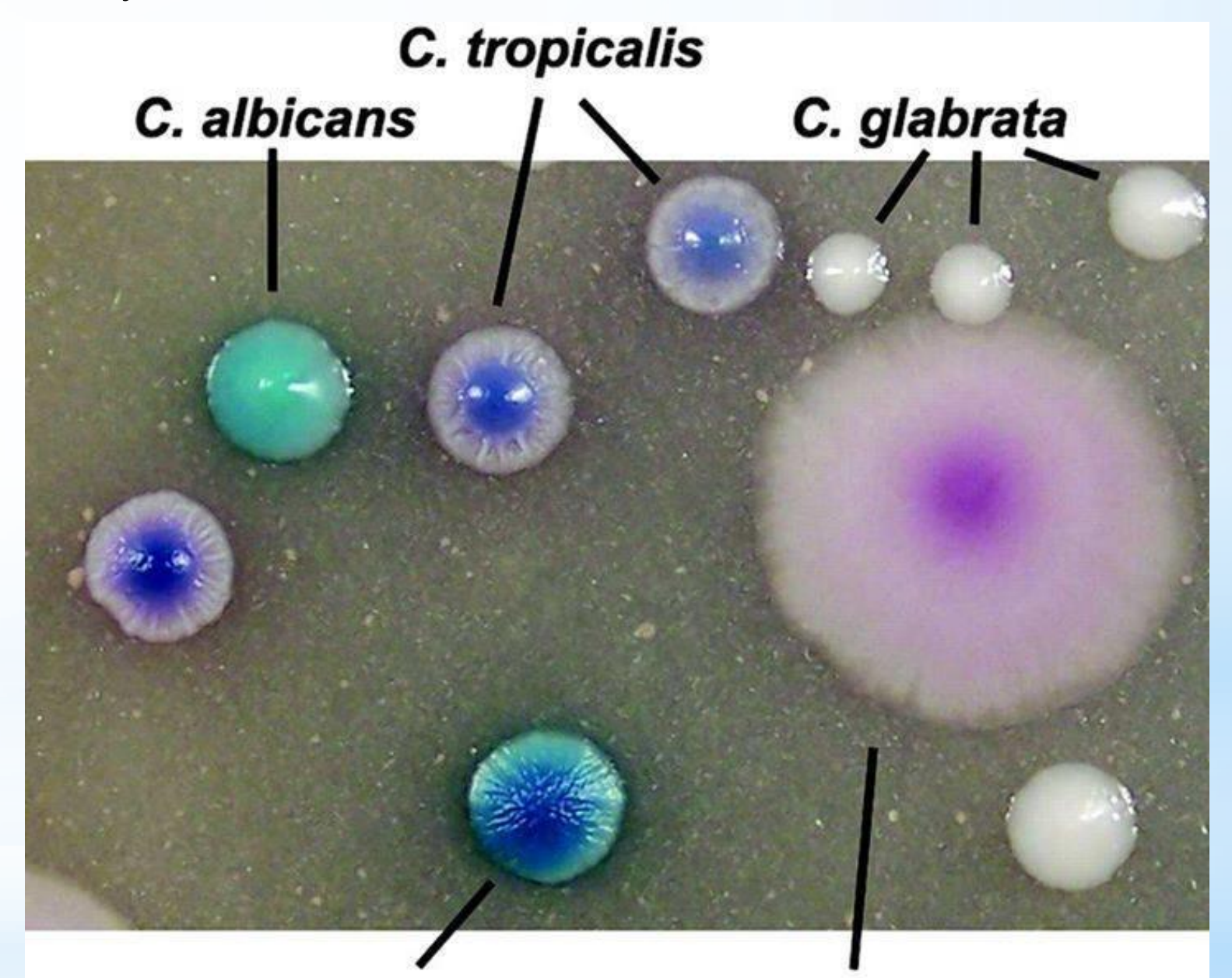

C. dubliniensis

C. krusei

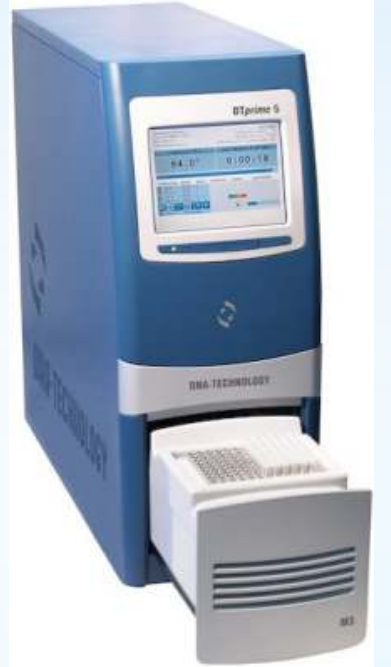

ATTTCCGCTAAAGCC

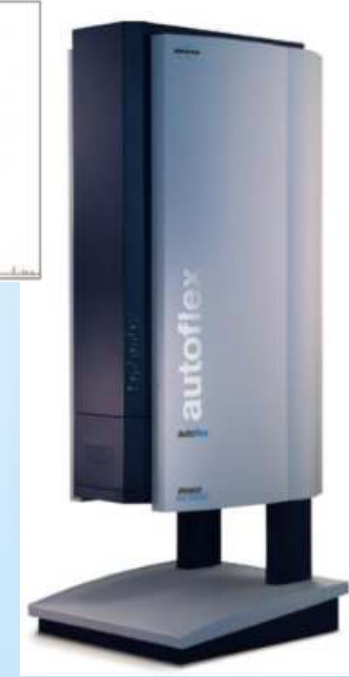

